\title{
Coulomb Breakup as a novel spectroscopic tool to probe directly the quantum numbers of valence nucleon of the exotic nuclei
}

\author{
Ushasi Datta Pramanik for s306 collaboration* \\ Saha Institute of Nuclear Physics, Kolkata 700064, India \\ E-mail: ushasi.dattapramanikesaha.ac.in
}

Coulomb breakup is a sensitive spectroscopic tool to probe ground state properties, particularly configuration with quantum numbers of loosely bound exotic nuclei. Due to large spatial extension of valence nucleon(s), enhanced electric dipole threshold strength of those nuclei can be observed through electromagnetic excitation. The observed threshold strength can be explained by a direct breakup mechanism. The shape and magnitude of these threshold strength of the exotic nuclei is a direct finger-print of the quantum numbers of valence nucleon and its occupation probability for that particular quantum state. Ground state properties of loosely bound p-s-d shell nuclei have been explored through kinematics complete measurement and this method has been established with certain limitation. Recently, using this method, an experiment (S306) has been performed to explore the ground state properties of a number of exotic $s d-p f$ shell neutron-rich nuclei around $(\mathrm{N} \sim 20)$ where first failure of magic number was reported. The experiment has been explored using LAND-ALADIN-FRS setup at energy 400-430 MeV/u. Invariant mass spectra of these nuclei have been obtained after measuring the four-momentum of all the decay products after one neutron removal using ${ }^{208} \mathrm{~Pb}$ target. The shape of the differential $\mathrm{CD}$ cross section suggests that the predominant ground-state configurations are ${ }^{28} \mathrm{Na}\left(1^{+}\right) \otimes v_{s, d},{ }^{29} \mathrm{Na}\left(3 / 2^{+}\right) \otimes v_{s, d}$ and ${ }^{34} \mathrm{Al}\left(4^{-}\right) \otimes v_{p}$ for ${ }^{29} \mathrm{Na}\left(3 / 2^{+}\right),{ }^{30} \mathrm{Na}\left(2^{+}\right)$and ${ }^{35} \mathrm{Al}\left(5 / 2^{+}\right)$isotopes, respectively. Comparison of our experimental findings with the shell model calculation using SDPM-M interaction suggests a lower limit of $s d-p f$ shell gap in ${ }^{30} \mathrm{Na}$. First time, very clear evidence of $p_{3 / 2}$ and $f_{7 / 2}$ shell inversion has been observed through the data analysis of Coulomb breakup of ${ }^{35} \mathrm{Al}$ $(\mathrm{N}=22)$. This results clearly indicate the breakdown of magic number $\mathrm{N}=28$. As it is evident from present experimental data of Coulomb dissociation of ${ }^{34} \mathrm{Al},{ }^{33} \mathrm{Mg}(\mathrm{N}=21)$, the ground state configuration is complicated and $\sim 50 \%$ core excited states contribution in the ground state of those isotopes are evident from experimental invariant mass spectra in coincidence with $\gamma$-rays. One may conclude that the sd-pf shell gap around $\mathrm{N} \sim 20$ is reduced and around $\sim 50 \%$ of the ground state configurations of these isotopes are multi particle-multi hole configurations across the shell gap. These isotopes may be considered as 'deformed halo' nuclei.

X Latin American Symposium on Nuclear Physics and Applications (X LASNPA),

1-6 December 2013

Montevideo, Uruguay

${ }^{*}$ Speaker. 


\section{Introduction}

100 years after discovery of the nucleus by Rutherford, the limits of the existence of the nuclei are still uncertain. Lack of detailed understanding of nucleon-nucleon interaction is main reason behind it. Experimental observation of the failure of validation of 'old magic number' in the nuclei near drip line further indicate that also. Recently, it has been notified that a number of ingredients in nucleon-nucleon interaction such as spin-isospin monopole interaction, three body interaction, tensor interaction part etc. are essential to explain experimentally observed properties of nuclei around drip line. The experimental data on nuclear shell structure around drip line is very essential and may provide important information on nucleon nucleon interaction. Investigation of nuclear shell structure around magic numbers are particularly important in this respect. 'Island of Inversion" are neutron-rich $\mathrm{Ne}, \mathrm{Na} . \mathrm{Mg}$ nuclei around N 20 and first failure of magic number had been reported in this region $[1,2]$. It has been observed that ground state properties of these nuclei can be explained only by considering pf shell contribution in addition to sd shell contribution and often, it has been concluded that these nuclei exhibit large deformation. Thus due to large deformation, sd-pf shell gap reduced. So, exploring ground state configuration with valence nucleon occupation probability will provide valuable information not only for nuclear structure but understanding of nucleon nucleon interaction what causes failure of magic numbers.

To probe the nuclear shell structure of loosely bound nuclei, a number of experimental methods with different reaction mechanism are being used by scientists. However, very few methods can probe directly the ground state configuration of loosely bound nuclei. These methods are Coulomb Breakup, knockout, transfer reaction etc. with certain limitations. The method Coulomb breakup is direct breakup of the nuclei into core and neutron by means of electromagnetic excitation at intermediate energy. In loosely bound nuclei, valence nucleon exhibit large spatial distribution which is decoupled from well bound normal density core. Through Coulomb breakup of loosely bound nuclei, enhanced dipole threshold strength can be observed due to large spatial distribution of valence neutron. The shape and strength of this threshold strength is sensitive to the quantum numbers of valence nucleon and its occupation probability. Thus this breakup is an exclusive spectroscopic tool for probing valence nucleon quantum states of loosely bound nuclei [3, 4]. In this article, some previous successful measurements will be mentioned to explain validity of the method at different situations. Later, preliminary new experimental results on nuclear shell structure of 'ISLAND of Inversion' nuclei, explored through Coulomb breakup will be presented.

\section{Coulomb breakup}

When projectile with relativistic energy passes by a high $\mathrm{Z}$ target it may be excited by absorbing virtual photons from the time dependent Lorentz-contracted Coulomb field [[5]] and breakup into core and neutron. This one neutron removal differential Coulomb dissociation cross section (CD) can be expressed by the following equation [3]:

$$
\frac{d \sigma_{c}}{d E^{*}}=\frac{16 \pi^{3}}{9 \hbar c} N_{E 1}\left(E^{*}\right) \Sigma_{j} C^{2} S\left(I_{c}^{\pi}, n l j\right) \times \Sigma_{m}|<q|(Z e / A) r Y_{m}^{l}\left|\psi_{n l j}(r)>\right|^{2}
$$

$\psi_{n l j}(r)$ and $<q \mid$ represent the single-particle wave function of the valence neutron in the projectile ground state and the final state wave-function of the valence neutron in the continuum respectively. 

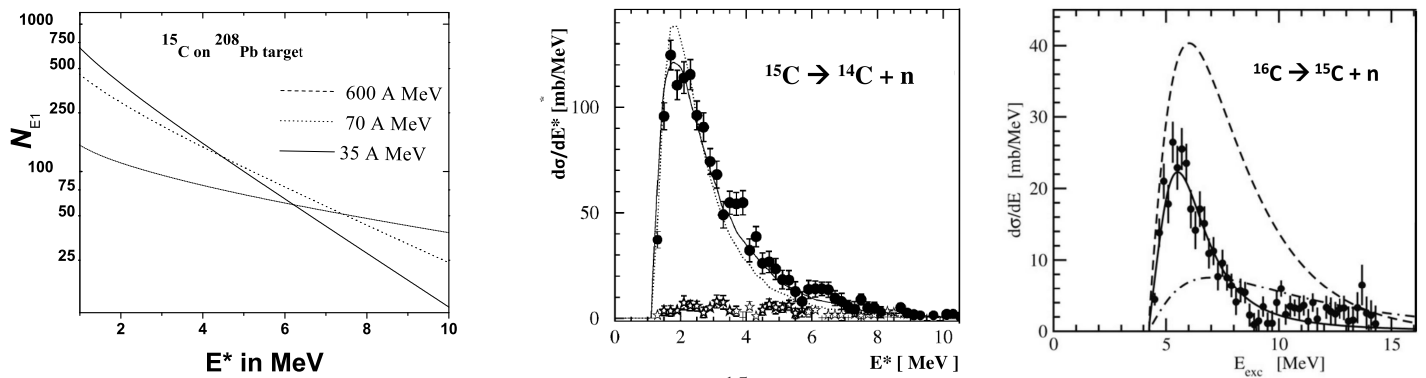

Figure 1: [left pannel] E1 vitual photon spectrum for ${ }^{15} \mathrm{C}$ projectile at $35 \mathrm{MeV} / \mathrm{u}, 70 \mathrm{MeV} / \mathrm{u}$ and $600 \mathrm{MeV} / \mathrm{u}$ on $\mathrm{Pb}$ target. [middle pannel] Differential Coulomb dissociation cross section with respect to excitation energy $\left(\mathrm{E}^{\star}\right.$ ) of ${ }^{15} \mathrm{C}$ breaking up into a neutron and a ${ }^{14} \mathrm{C}$ fragment in its ground state (filled circles). The solid curve displays the result from the direct-breakup model in plane-wave approximation and valence neutron is occupying s-wave. Figure reprinted from Datta Pramanik et al. Copyright (2003), with permission from Elsevier [right panel] Differential Coulomb dissociation cross section with respect to excitation energy $\left(\mathrm{E}^{\star}\right.$ ) of ${ }^{16} \mathrm{C}$ breaking up into a neutron and a ${ }^{15} \mathrm{C}$ fragment in its ground state (filled circles). The solid curve displays the result from the direct-breakup model in plane-wave approximation. Figure reprinted from Datta Pramanik et al. Copyright (2007), with permission from Elsevier.

$C^{2} S\left(I_{c}^{\pi}, n l j\right)$ represents the spectroscopic factor with respect to a particular core state $I_{c}^{\pi}$ of the valence neutron in the projectile ground state. $N_{E 1}\left(E^{\star}\right)$ is the number of virtual photon as a function of excitation energy $E^{\star}$ which can be computed in a semi-classical approximation [5]. Figure 1, [left panel] shows E1 virtual photon spectrum for ${ }^{15} \mathrm{C}$ projectile at $35 \mathrm{MeV} / \mathrm{u}, 70 \mathrm{MeV} / \mathrm{u}$ and $600 \mathrm{MeV} / \mathrm{u}$ on $\mathrm{Pb}$ target. It is evident from virtual photon spectra that number of electric dipole virtual photon $\left(N_{E 1}\right.$ decrease with excitation energy $\left(\mathrm{E}^{\star}\right)$ for low energy projectile due to adiabatic cutoff. Figure 1 [right panel] shows differential Coulomb dissociation cross section with respect to excitation energy $\left(\mathrm{E}^{\star}\right)$ of ${ }^{15} \mathrm{C}$ breaking up into a neutron and a ${ }^{14} \mathrm{C}$ fragment in its ground state (filled circles). The solid curve displays the result from the direct-breakup model considering valence neutron occupation probability in $\mathrm{s}_{1 / 2}$ orbital and after breakup that neutron in continuum can be presented by plane-wave approximation. As it is evident from the figure 1 that this plane wave approximation is in agreement with the data of ${ }^{15} \mathrm{C}$ breakup. But the situation for ${ }^{16} \mathrm{C}[6]$ and ${ }^{23} \mathrm{O}$ [7] like more deeply bound neutron-rich nuclei is different. Figure 2. Differential Coulomb dissociation cross section with respect to excitation energy $\left(E^{\star}\right)$ of ${ }^{16} \mathrm{C}$ breaking up into a neutron and a ${ }^{15} \mathrm{C}$ fragment in its ground state (filled circles). The dashed and solid curve displays the result from the direct-breakup model calculation considering neutron in $\mathrm{s}_{1 / 2}$ orbital in ground state of that isotope going to continuum which is represented by plane-wave approximation and distorted wave respectively. Clearly, more deeply bound cases i,e ${ }^{16} \mathrm{C},{ }^{23} \mathrm{O}$, neutron in continuum can be presented well by distorted wave due to final state interaction. [right panel] sum-energy spectrum of $\gamma$-decay transitions from ${ }^{16} \mathrm{C}$ after Coulomb breakup of ${ }^{17} \mathrm{C}$. The inset shows a partial scheme of levels in ${ }^{16} \mathrm{C}$ and their population after Coulomb breakup. Thus, it is important to measure $\gamma$-rays of breakup core in coincidence with breakup neutron. The ground state spin and parity of exotic nuclei can be obtained by coupling spin and parity of core state with that of valence neutron.

\section{Experiment}

Short-lived radioactive nuclei were produced by the fragmentation of the primary beam ${ }^{40} \mathrm{Ar}$ 

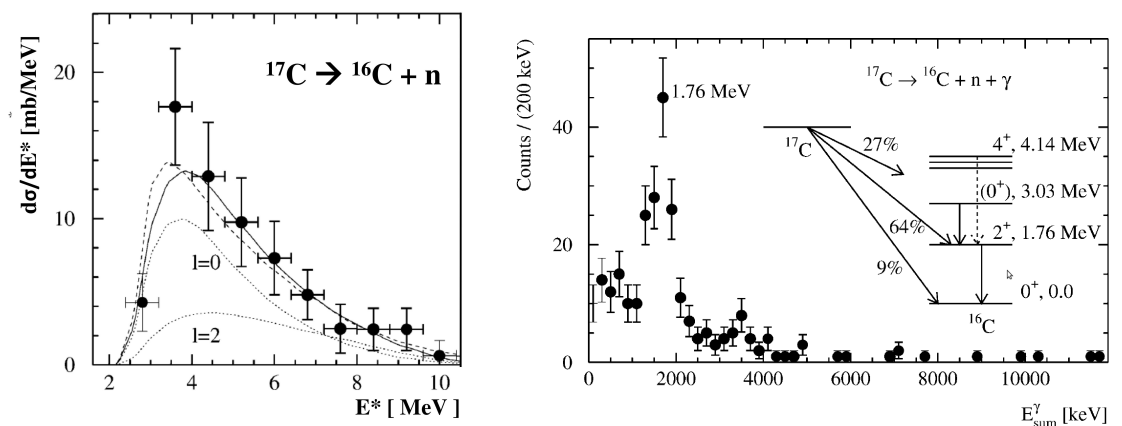

Figure 2: [Left pannel] Differential Coulomb dissociation cross section with respect to excitation energy $\left(\mathrm{E}^{\star}\right.$ ) of ${ }^{17} \mathrm{C}$ breaking up into a neutron and a ${ }^{16} \mathrm{C}$ fragment in its first excitted state (filled circles). The solid curve displays the result from the direct-breakup model in plane-wave approximation. [right pannel] sum-energy spectrum of $\gamma$-decay transitions from ${ }^{16} \mathrm{C}$ after Coulomb breakup of ${ }^{17} \mathrm{C}$. The inset shows a partial scheme of levels in ${ }^{16} \mathrm{C}$ and their population after Coulomb breakup. Figure reprinted from Datta Pramanik et al. Copyright (2003), with permission from Elsevier

at $530 \mathrm{MeV} / \mathrm{u}$ on a ${ }^{9} \mathrm{Be}$ production target $\left(8 \mathrm{gm} / \mathrm{cm}^{2}\right)$ at GSI, Darmstadt. The secondary beams, $\left({ }^{29-31} \mathrm{Na},{ }^{31-33} \mathrm{Mg},{ }^{34,35} \mathrm{Al}\right.$ etc.) with similar mass to charge ratio (A/Z between 2.55 to 2.85$)$ as shown in Fig. 1(a) were separated according to the magnetic rigidities by the fragment separator (FRS). The secondary cocktail beam was bombarded on lead $\left(2 \mathrm{gm} / \mathrm{cm}^{2}\right)$ and carbon $\left(935 \mathrm{mg} / \mathrm{cm}^{2}\right.$ target for studying electromagnetic and nuclear excitation, respectively. In order to detect the $\gamma$-rays from the excited projectile or projectile like fragments, the reaction target was surrounded by the 4 П-crystal ball spectrometer which consists of $162 \mathrm{NaI}$ crystals. Eight double-sided Silicon Strip Trackers (SSTs) were placed enclosing the reaction target in $4 \Pi$ solid angle. After reaction at the secondary target, reaction fragments and neutron(s) are forward focused due to Lorentz boost and passes through A Large Dipole Magnet (ALADIN). Reaction fragments, deflected by ALADIN according to their $\mathrm{A} / \mathrm{Z}$ ratios, were tracked by two large scintillating fiber detectors (GFIs) and detected by the time of flight wall (TFW). The trajectories of neutrons remain unchanged and were detected by the Large Area Neutron Detector (LAND). Data analysis has been performed using CERN-ROOT platform and program developed at GSI, Darmstadt and SINP, Kolkata $[8,9]$.
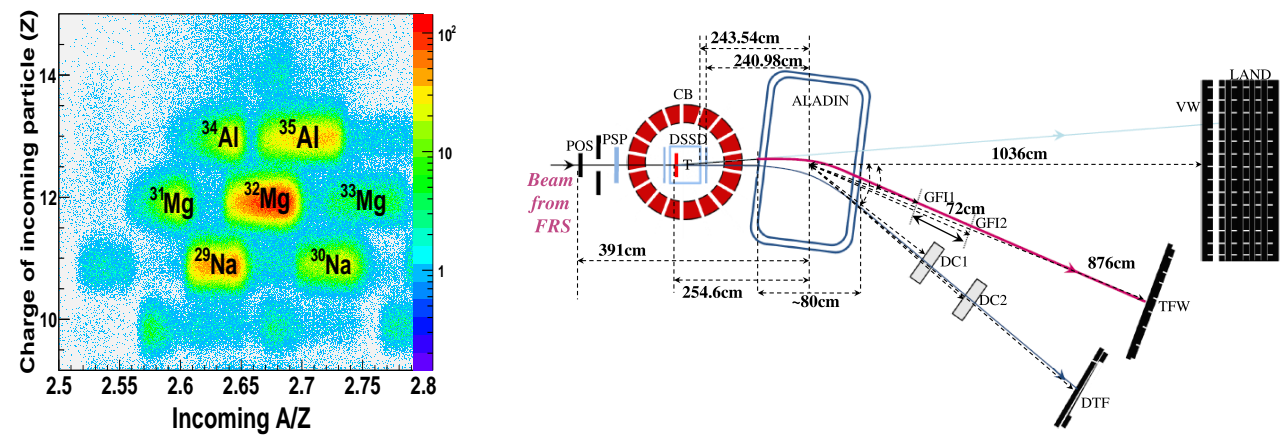

Figure 3: [Left panel] Coctail beam .[right panel] Schematic diagram of experimental setup of experiment S306 at GSI, Darmstadt 


\section{Analysis and Result}

By measuring the four-momenta $\mathrm{P}_{i}$ of all the decay products, the excitation energy $\mathrm{E}^{*}$ of the nucleus prior to decay can be reconstructed on an event-by-event basis by analyzing the invariant mass M. The Coulomb dissociation cross section measured with the ${ }^{208} \mathrm{~Pb}$ target $\left(2 \mathrm{gm} / \mathrm{cm}^{2}\right)$ was obtained after subtracting nuclear contributions determined from the data obtained with a ${ }^{12} \mathrm{C}$ target $\left(0.935 \mathrm{gm} / \mathrm{cm}^{2}\right)$ and applying a proper scaling of the cross sections. Background contributions from reactions induced by materials of detectors were determined from data taken without any target and were subtracted.

The Coulomb breakup of ${ }^{29,30} \mathrm{Na}$ and ${ }^{35} \mathrm{Al}$ yield mainly (70-80\%) core in ground state. Figure 4. shows [left panel] differential Coulomb dissociation cross section with respect to excitation energy ( $\mathrm{E}$ ) of ${ }^{29} \mathrm{Na}$ breaking into core ${ }^{28} \mathrm{Na}$ and neutron. The solid line represents the direct breakup model calculation where valence neutron is in $s$ and $d$-wave. Right panel of figure 4 . shows differential Coulomb dissociation cross section with respect to excitation energy (E ) of ${ }^{35} \mathrm{Al}$ breaking into core ${ }^{34} \mathrm{Al}$ and neutron. The solid line represents the direct breakup model calculation where valence neutron is occupying p-orbital. Comparison of experimental CD crosssection with direct breakup model calculation favors ${ }^{34} \mathrm{Al}(\mathrm{g} . s) \otimes v_{p_{3 / 2}}$ as ground state configuration of ${ }^{35} \mathrm{Al}$. Monte-Carlo shell model calculation by Otsuka et. al. [10] has predicted the lowering of $2 \mathrm{p}_{3 / 2}$ orbital for aluminum isotopes around the $\mathrm{N} \sim 20$ shell gap. On the otherhand the shape of the differential Coulomb dissociation cross section and comparison with direct brakup model calculation suggest the predominant ground state as ${ }^{28} N a\left(1^{+}\right) \otimes v_{s, d}$ and ${ }^{29} N a\left(3 / 2^{+}\right) \otimes v_{s, d}$ for ${ }^{29} \mathrm{Na}$ and ${ }^{30} \mathrm{Na}$ isotope, respectively. In table 1 the experimental total Coulomb dissociation cross sections of neutron-rich nuclei upto $10 \mathrm{MeV}$ excitation have been presented. The corresponding direct breakup model calculation results are compared with the experimental findings. It may be noted that valence neutron binding energy ${ }^{30} \mathrm{Na},{ }^{33} \mathrm{Mg},{ }^{34} \mathrm{Al}$ are close to each other. But the total $\mathrm{CD}$ cross-sections are very different which indicate different configurations in ground state of those isotopes. Similar type of observation also reported [11] in knockout reaction.

The ground state configurations of ${ }^{34} \mathrm{Al}$ and ${ }^{33} \mathrm{Mg}(\mathrm{N}=21)$ isotope are more complicated. It is evident from observed $\gamma$-ray spectra of ${ }^{33} \mathrm{Al}$ and ${ }^{32} \mathrm{Mg}$ obtained after Coulomb breakup that around $\sim 50 \%$ of ground state configuration are with core in excited states. One may conclude that the sd-pf shell gap around $\mathrm{N} \sim 20$ is reduced and around $\sim 50 \%$ of the ground state configurations of these isotopes are multi particle-multi hole configurations across the shell gap. For ${ }^{34} \mathrm{Al}$, this has been also reported through $\mathrm{g}$ factor measurement [12]. For ${ }^{33} \mathrm{Mg}$ both beta decay measurement [13] and magnetic moment measurement [14] favors ground state configuaration as multi particle-hole configuration. Though parity of the ground state configuration of this isotope is in contradiction. These isotopes may be considered as 'deformed halo' nuclei. The shape of CD cross-section is not in agreement with the configuration as ${ }^{32} M g\left(0^{+}\right) \otimes v_{p 3 / 2}$ as predicted by knockout reaction [15]. The shape of CD cross-section with core excited state is in agreement with s wave. More details investigation is going on.

\section{Conclusion}

Our new experimental results clearly demonstrate that Coulomb breakup is even successful if heavier nuclei at sd-pf shell. The limitation of this method is that the sensitivity of tail part of the 

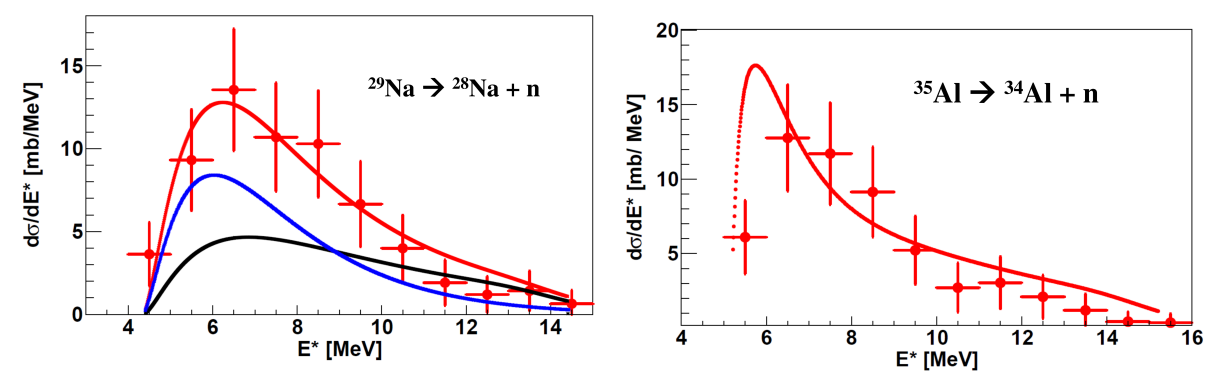

Figure 4: Differential Coulomb dissociation cross section with respect to excitation energy (E ) of ${ }^{29} \mathrm{Na}$ breaking up into a neutron and a ${ }^{28} \mathrm{Na}$ fragment in its ground state (filled circles). The solid curve displays the result from the direct-breakup model in plane-wave approximationand valence neutron is in $s, d$ orbital. [right pane] Differential Coulomb dissociation ${ }^{35} \mathrm{Al}$ cross section with respect to excitation energy (E) of breaking up into a neutron and a ${ }^{34} \mathrm{Al}$ fragment in its ground state (filled circles). The solid curve displays the result from the direct-breakup model in plane-wave approximation and valence neutron is occupying $p$ orbital.

Table 1: Coulomb dissociation cross-section of ${ }^{35,34} \mathrm{Al},{ }^{33} \mathrm{Mg},{ }^{30,29} \mathrm{Na}$ nuclei up to $10 \mathrm{MeV}$ excitation energy

\begin{tabular}{|c|c|c|c|c|c|}
\hline Isotope & $\begin{array}{c}\text { ground state } \\
\text { spin } \\
\text { and parity }\end{array}$ & $\begin{array}{c}S_{n} \\
(\mathrm{MeV})\end{array}$ & $\begin{array}{c}\text { neutron } \\
\text { orbital }\end{array}$ & $\begin{array}{l}\text { Direct } \\
\text { Breakup } \\
\text { model }\end{array}$ & $\begin{array}{l}\text { Cross-section } \\
\qquad(\mathrm{mb}) \\
\text { Experiment }\end{array}$ \\
\hline${ }^{35} A l$ & $\left(5 / 2^{+}\right)$ & 5.2 & $\begin{array}{l}p_{3 / 2} \\
f_{7 / 2}\end{array}$ & $\begin{array}{c}42 \\
3\end{array}$ & $72 \pm 10$ \\
\hline${ }^{34} \mathrm{Al}$ & $\left(4^{-}\right)$ & 2.68 & $\begin{array}{l}p_{3 / 2} \\
f_{7 / 2}\end{array}$ & $\begin{array}{c}150 \\
20\end{array}$ & $70 \pm 10$ \\
\hline${ }^{33} \mathrm{Mg}$ & $\begin{array}{c}\left(3 / 2^{-}\right) \text {or } \\
\left(3 / 2^{+}\right)\end{array}$ & 2.27 & $\begin{array}{l}p_{3 / 2} \\
f_{7 / 2} \\
s_{1 / 2}\end{array}$ & $\begin{array}{c}167 \\
3 \\
215\end{array}$ & $101 \pm 10$ \\
\hline${ }^{30} \mathrm{Na}$ & $2^{+}$ & 2.37 & $\begin{array}{l}s_{1 / 2} \\
d_{5 / 2}\end{array}$ & $\begin{array}{c}215 \\
56\end{array}$ & $145 \pm 12$ \\
\hline${ }^{29} \mathrm{Na}$ & $3 / 2^{+}$ & 4.4 & $\begin{array}{l}s_{1 / 2} \\
d_{5 / 2}\end{array}$ & $\begin{array}{l}89 \\
22\end{array}$ & $90 \pm 7$ \\
\hline
\end{tabular}

wave functions of valence nucleon,. Hence this method is useful for valence nucleon occupying low-1 orbital of loosely bound nuclei. It has been observed that for more deeply bound nuclei, final state of interaction is important.

The observed low-lying dipole strength in these neutron-rich $\mathrm{Na}, \mathrm{Mg}, \mathrm{Al}$ isotopes can be understood as a direct-breakup mechanism and no resonance like structure has been observed.

The shape of the differential CD cross section suggests that the predominant ground-state configurations are ${ }^{28} N a\left(1^{+}\right) \otimes v_{s, d}$ and ${ }^{29} N a\left(3 / 2^{+}\right) \otimes v_{s, d}$ for ${ }^{29} N a\left(3 / 2^{+}\right)$and ${ }^{30} N a\left(2^{+}\right)$isotopes, 
respectively. These ground state configurations of the neutron-rich $\mathrm{Na}$ isotopes $(\mathrm{N}=18,19)$ support previous reported ground state spin and parity of these nuclei. The experimental observation for ${ }^{35} \mathrm{Al}$ is different. Comparison of experimental CD cross-section with direct breakup model calculation with neutron in $p_{3 / 2}$ orbital favors ${ }^{34} A l(g . s) \otimes v_{p_{3 / 2}}$ as ground state configuration of ${ }^{35} \mathrm{Al}$. This clearly shows failure of magic number at $\mathrm{N}=28$. First time, very clear evidence of $p_{3 / 2}$ and $f_{7 / 2}$ shell inversion has been observed through the data analysis of Coulomb breakup of ${ }^{35} \mathrm{Al}(\mathrm{N}=22)$. This results clearly point the breakdown of magic number $\mathrm{N}=28$ and may be indication of new magic number.

\section{Acknowledgement}

The data and experimental results which has been presented here is an effort of large collaboration, S306 (GSI expt.) Author acknowledges her collaborators, Mr. A. Rahaman, Mr. S. Chakraborty from SINP, KOlkata; Prof. T. Aumann, Dr. K. Boretzky, Prof.H. Emling, Prof. G. Muenchenberg, Dr. H. Simon, Dr. H. Weick from GSI, Darmstadt and all other S306 collaborators. Author is thankful to Alexander von Humboldt foundation for their support during proposing, defending and performing this experiment (S306) at GSI Darmstadt

\section{References}

[1] C. Thibault, et al., Phys. Rev. C 12 (1975) 644

[2] T. Motobayashi, et al., Phys. Lett. B 346 (1995) 9

[3] U. Datta Pramanik, et al., Phys. Lett. B 551 (2003) 63

[4] T. Nakamura, et al., Phys. Rev. Lett. 83 (1999) 1122

[5] C.A.Bertulani and G.Baur, Physics Report 163 (1988) 299

[6] U. Datta Pramanik, Prog. in Particle and Nucl. Phys. 59 (2007) 183

[7] C. Nociforo, et. al., Phys. Lett. B 605 (2005) 79

[8] S. Chakraborty, et. al., EPJ 66, (2014) 02019

[9] A. Rahaman, et al., EPJ Web of Conferences 66, (2014) 02087

[10] T. Otsuka, et al., Phys. Rev. Lett. 87 (2001) 082502; Phys. Rev. Lett. 104 (2010) 012501

[11] C.Rodriguez-Tajes, et al., Phys. Rev. C (2010) 024305

[12] P.Himpe et al., Phys. Lett. B 658 (2008) 203

[13] V. Tripathi, et al., Phys. Rev. Lett. 94 (2008) 142504

[14] D.T.Yordanov et al, Phys. Rev. Lett. 99 (2007) 212501

[15] R.Kanungo et. al, , Phys. Lett. B 685 (2010) 253 